\section{The distance learning on headache disorders and pain}

Today, we are observing a rapid development of medical culture and, particularly for what concerns our readership, there is a growing body of congresses, (e-)papers and (e-)books devoted to the discussion of various headaches and pain conditions. Among the aspects of headache and pain most debated in the new media, we find the genetics and pathophysiology of migraine, the emerging socio-economic data on headaches, the clinical use of innovative drugs for migraine, the application of existing drugs to chronic tension headache, the availability of new non-narcotic analgesics, and the use of new technologies in the management of chronic pain. This information is rapidly diffused through the Web and directly reaches the desktop of doctors involved in research and in patient management in this broad area of medicine. However, the amount and variability of the documentation published online requires that we have an organized approach to collecting such information from the Web. Research using generic search engines usually provides an overwhelming number of Web sites of dubious usefulness, at least for the inexperienced searcher, and the selection of quality sites can be unsatisfying and inefficient.

\section{Medscape from WebMD}

The ultimate goal of a medical Web site is provide clinicians and other healthcare professionals the most timely sources of information relevant to their patients and practices. The additional goal is to make the task of clinical information gathering simpler, more fruitful, and less time-consuming. These goals are achieved by the medical information Web site "Medscape" from WebMD (http://www.medscape.com/cmecenterdirectory/).

This site offers specialists as well as primary care physicians integrated medical information and education tools. After a simple, one time, free registration, Medscape automatically delivers the specialty site that best fits the user's profile. Registered users can choose from a variety of specialty interfaces to Medscape, and for physicians interested in headaches, these may be internal medicine, neurology and neurosurgery, pharmacotherapy, primary care, rheumatology, or family medicine. The internal search engine of Medscape is simple to use and helps refine searching. The exhaustive content available on Medscape includes the latest news about congresses (in Conference Coverage), Highlights from Resource Centers, selected journal articles and news from international media networks. The regular use of this medical Web site can support our updating activities in the field of headache and pain. 